\title{
Anti-angiogenic and Immunomodulatory Effect of the Herbal Medicine "Juzen-taiho-to" on Malignant Glioma
}

\author{
Hiroshi Kamiyama, Shingo Takano, * Eiichi Ishikawa, Koji Tsuboi, and Akira Matsumura \\ Department of Neurosurgery, Institute of Clinical Medicine, Graduate School of Comprehensive Human Sciences, \\ University of Tsukuba; 1-1-1 Tennoudai, Tsukuba, Ibaraki 305-8575, Japan. \\ Received May 23, 2005; accepted August 11, 2005; published online August 19, 2005
}

\begin{abstract}
Juzen-taiho-to (JTT) is known as a Japanese herbal medicine that increases the immune function via the enhancement of phagocytosis, cytokine induction, and antibody production. Anti-neoplastic effects on malignant gliomas have been reported by means of the enhancement of the immune function in both animals and humans. We evaluated whether JTT has anti-angiogenic effects on malignant glioma growth in vitro and in vivo. In vitro, the anti-proliferative effect of JTT on malignant glioma cells and endothelial cells was assessed by cell proliferation assay. In vivo, a subcutaneous model of malignant glioma with different-aged mice (old, 43 weeks; young, 8 weeks) was used. After oral administration of JTT to mice, their immunological function and angiogenic status of tumor tissues were assessed by flow cytometry and immunohistochemistry, respectively. JTT inhibited human endothelial cell, but not glioma cell, proliferation in vitro. In vivo, the NK (natural killer) cell ratio within PBMC (peripheral blood mononuclear cells) and NK activity of fresh splenocytes obtained from JTT-treated old mice were significantly increased compared to the ratio and activity in control mice. In old mice, the vessel area of tumor tissues in JTT treatment groups was significantly decreased. These enhancements of immunological function and the inhibition of angiogenic activity were not observed in young mice. JTT not only increased host immunological function but also exerted anti-angiogenic effects on malignant glioma growth. JTT would be useful as an adjuvant medicine for malignant gliomas through its enhancement of systemic immunological function and its anti-angiogenic action.
\end{abstract}

Key words Juzen-taiho-to; anti-angiogenesis; glioma; natural killer (NK) activity; endothelial cell; vascular endothelial growth factor (VEGF)

Angiogenesis, the growth of new blood vessels pre-existing in tissue, is crucial to glioma growth, and malignant glioma is characterized by its invasiveness and angiogenesis. In fact, glioblastoma is one of the most richly neovascularized solid tumors in terms of vasoproliferation, endothelial hyperplasia, and endothelial cytology. Therefore, anti-angiogenic treatment represents a promising therapeutic modality for gliomas. ${ }^{1,2)}$

Herbal medicines (Japanese and Chinese traditional medicines) are attracting attention as a treatment method for patients with solid tumors. ${ }^{3,4)}$ Especially, Juzen-taiho-to (JTT), which consists of 10 component herbs, is known to improve the general systemic condition of cancer patients by reducing the adverse effects of chemotherapy, radiation therapy, and surgical treatment ${ }^{4,5)}$ and to inhibit metastases. ${ }^{6,7)}$ The mechanisms of these anti-neoplastic effects of JTT have been generally explained through various immuno-biological activities, including the enhancement of natural killer (NK) and natural killer T (NKT) cell activity, elevation of interferon- $\gamma$ (IFN- $\gamma$ ) production, and anti-tumor effects. ${ }^{8-10)}$

We investigated the anti-angiogenic as well as systemic immunological activity of JTT for young and old mice with malignant gliomas because (1) some interactions between immune function and angiogenesis have been reported, ${ }^{11,12)}$ (2) herbal medicine is orally available for a long period without prominent side effects, and (3) the anti-angiogenic effects of herbal medicine have been investigated only in limited studies. ${ }^{13,14)}$

\section{MATERIALS AND METHODS}

Preparation of Herbal Medicine The herbal medicine,
Juzen-taiho-to (JTT) (TJ-48), used in this study was obtained from Tsumura \& Co., Ltd. (Tokyo). JTT was prepared as a spray-dried powder of hot water extract obtained from ten medical plants in the ratio of Astragali Radix (3.0 g), Cinamomi Cortex (3.0 g), Rehmanniae Radix (3.0 g), Paeoniae Radix (3.0 g), Cnidii Rhizoma (3.0 g), Angelicae Radix $(3.0 \mathrm{~g})$, Ginseng Radix $(3.0 \mathrm{~g})$, Hoelen $(3.0 \mathrm{~g})$, Glycyrrhizae Radix (1.5 g) [,] and Atractylodis Lanceae Rhizoma (3.0 g). The JTT diet feed of mixing low-dose $(0.5 \%)$ or high-dose (1\%) JTT extracts was made by the Oriental Yeast Co., Ltd. (Tokyo).

Cell Lines A human glioma cell line, U87-MG, calf pulmonary artery endothelial cell line, CPAE, and NK-sensitive murine lymphoma cell line, YAC-1, were obtained from the American Type Culture Collection (Rockville, MD, U.S.A.). A mouse glioma cell line, GL261, was a gift from Dr. Masahiro Toda (Keio University). An immortalized human umbilical vein endothelial cell line, TE-1, was a gift from Dr. Youji Mitsui (Tokushima Bunri University). ${ }^{15)}$ U87-MG, GL261, TE-1, and CPAE were maintained in Eagle's minimum essential medium supplemented with $10 \%$ fetal calf serum (FCS) and 5\% penicillin-streptomycin solution (Sigma, St. Louis, MO, U.S.A.). YAC-1 was maintained in RPMI 1640 medium supplemented with 10\% FCS and 5\% penicillin-streptomycin solution. At each passage, cells were harvested as single-cell suspensions using trypsin/EDTA. U87-MG, GL261, and YAC-1 were cultured in a falcon flask (Becton Dickinson, Franklin, NJ, U.S.A.). TE-1 and CPAE were cultured in a collagen type I coated flask (IWAKI, Tokyo, Japan). Cells were incubated in a humidified atmosphere containing $5 \% \mathrm{CO}_{2}$ at $37^{\circ} \mathrm{C}$.

Preparation of the JTT Mixture Medium JTT extract 
was mixed with Eagle's minimum essential medium supplemented with $10 \%$ FCS and 5\% penicillin-streptomycin solution to a concentration of $2.5 \mathrm{mg} / \mathrm{ml}$. After the mixture was shaken at $37^{\circ} \mathrm{C}$ for $1 \mathrm{~h}$ in a water bath, it was centrifuged at $9000 \mathrm{rpm}$ for $15 \mathrm{~min}$ to remove insoluble materials. Then the supernatant was sterilized by filtration with a disposable syringe filter, the Acrodisc Syringe Filter with a $0.2-\mu \mathrm{m}$ membrane (Pall Corporation, MI, U.S.A.). This solution was used in the analysis of the cell proliferation assay.

Cell Proliferation Assay (WST-8 Assay) and Cell Viability Assay (Trypan Blue Dye Exclusion Assay) The inhibition of cell proliferation was determined by a WST-8 (2-(2-methoxy-4-nitrophenyl)-3-(4-nitrophenyl)-5-(2,4-disulfophenyl)-2 $H$-tetrazolium, monosodium salt) assay kit (Kishida Kagaku, Osaka, Japan). The kit results are based on the conversion of the tetrazolium salt WST- 8 to highly watersoluble formazan by viable cells. Briefly, cells $\left(5 \times 10^{3}\right.$ cells/well) in 96-well plates were incubated overnight. Then, the medium was changed to the new medium with various concentrations $(0,0.1,0.5,1,1.75,2.5 \mathrm{mg} / \mathrm{ml})$ of JTT solution. After $48 \mathrm{~h}$ of incubation, WST- 8 reagents were added to the culture. After $2 \mathrm{~h}$ of incubation, the absorbance at $450 \mathrm{~nm}$ was measured with a Model 550 microplate reader (BioRad, Hercules, CA, U.S.A.). Absorbance correlates with the viability of cells. Cell number ( $\%$ of control) was calculated with the following formula: cell number $(\%$ of control $)=$ (each absorbance-absorbance of blank well)/(absorbance of $0 \mathrm{mg} / \mathrm{ml}$ well $) \times 100$.

Cell viability to JTT extract was determined by a trypan blue dye exclusion assay. Cells $\left(5 \times 10^{4}\right.$ cells/well $)$ in 24 -well plates were incubated overnight. Then, the medium was changed to the new medium with various concentrations $(0$, $0.1,0.5,1,1.75,2.5 \mathrm{mg} / \mathrm{ml}$ ) of JTT solution. After $48 \mathrm{~h}$ of incubation, cell suspension was mixed with equal volume of $0.3 \%$ trypan blue aqueous solution (Wako, Osaka, Japan). Cells were observed under a microscope and counted viable cells on hemocytometer. Cell viability (\%) was calculated with the following formula: cell viability $(\%)=($ number of viable cells/number of total cells) $\times 100$.

All experiments were performed in triplicate.

Mice Female C57BL/6 mice were purchased from Japan Clea (Tokyo). In this study, young mice were 8 weeks of age and old ones were 43 weeks of age. These mice were maintained under a constant temperature, humidity, and light-controlled environment with free access to food and water.

GL261 C57BL/6 Mouse Subcutaneous Model Three weeks after the implantation of $1 \times 10^{7}$ GL261 cells in the flank of an 8-week-old C57BL/6 mouse, GL261 tumor tissue fragments were removed and then harvested tumor fragments $1 \mathrm{~mm}^{3}$ in size were implanted in the flanks of other young and old mice. These young and old mice were separated into three groups: high-dose JTT (1000 mg/kg/d JTT), low-dose JTT $(500 \mathrm{mg} / \mathrm{kg} / \mathrm{d}$ JTT), and MF feed as a control (Oriental Yeast, Tokyo). The volume of MF feed eaten per day was measured prior to the experiment, and the volume of the mixture of JTT powder was calculated based on these experiments. After the tumor implantation, the mice in the JTT groups were fed the diet containing JTT for 3 weeks.

Measurement of Tumor Volume and Mice Weight The size of the tumors was measured three times in a week by caliper, and the weight of mice was measured before and after JTT treatment. Tumor volume $(T V)$ was calculated using the formula: $T V=W^{2} \times L / 2$ (with $W$ being the shortest diameter and $L$ being the longest diameter). Three weeks after the implantation, mice were sacrificed by an overdose of ethyl ether. Immediately, the subcutaneous tumor and spleen were harvested from each mouse.

Flow Cytometry The spleen obtained from each mouse was minced in RPMI 1640 medium and separated with a cell strainer of $70 \mu \mathrm{m}$. The cell suspensions were washed with buffer containing $150 \mathrm{~mm} \mathrm{NH}{ }_{4} \mathrm{Cl}, \mathrm{pH} 7.2,0.01 \mathrm{~mm} \mathrm{KHCO}_{3}$, $0.1 \mathrm{~mm}$ 2Na-EDTA, and RPMI 1640 medium containing $15 \mathrm{~mm} \mathrm{NaHCO}, 100 \mathrm{U} / \mathrm{ml}$ penicillin $\mathrm{G}, 100 \mu \mathrm{g} / \mathrm{ml}$ streptomycin, $50 \mu \mathrm{M} 2$-mercaptoethanol, and $10 \mathrm{~mm}$ HEPES. The suspensions were centrifuged at $1500 \mathrm{rpm}$ for $5 \mathrm{~min}$ at $4{ }^{\circ} \mathrm{C}$. All reagents used to stain the cell suspensions with antibodies were purchased from BD Pharmingen (San Diego, CA, U.S.A.). After staining with anti-Fc receptor antibody $2.4 \mathrm{G} 2$, each sample $\left(1 \times 10^{6}\right.$ cells $)$ was stained with FITCanti-CD3e (145-2C11), PE-anti-NK-1.1 (PK136), FITC-antiCD4 (GK1.4), and PE-anti-CD8a (53-6.7) for $30 \mathrm{~min}$ at $4{ }^{\circ} \mathrm{C}$. Each sample was washed with PBS for flow cytometric analysis. Each cell percentage was measured on a FACS Calibur flow cytometer equipped with Cell Quest software (BD, Mountain View, CA, U.S.A.).

NK Activity NK activity of fresh splenocytes was measured using WST-8 reagent ${ }^{16)}$ (Kishida Chemical Co., Inc., Osaka, Japan). A volume of $50 \mu \mathrm{l}$ of the target cell suspension (YAC- 1 cells, $1 \times 10^{5}$ cells/well) was added to $50 \mu \mathrm{l}$ of the effector cells (fresh nonactivated spleen cells) at different effector : target $(\mathrm{E}: \mathrm{T})$ ratios $(5: 1,20: 1$, and $30: 1)$ into 96well flat-bottomed culture plates. The plates were centrifuged at $1000 \mathrm{rpm}$ for $5 \mathrm{~s}$ and then incubated in a $5 \% \mathrm{CO}_{2}$ incubator at $37^{\circ} \mathrm{C}$. After $4 \mathrm{~h}$ of incubation, $10 \mu \mathrm{l}$ of WST- 8 reagent was added, and then the plates were additionally incubated for 1 h. Then, the optical density (OD) was determined in a microplate spectrophotometer at $450 \mathrm{~nm}$ (reference $655 \mathrm{~nm}$ ). The cytotoxic activity in each $\mathrm{E}: \mathrm{T}$ ratio was calculated as follows: cytotoxicity (NK activity) $\%=(\mathrm{OD}$ target $-(\mathrm{OD}$ target + effector $-O D$ effector $)-O D$ blank $) /(O D$ target-OD blank) $\times 100$.

Immunohistochemistry for Angiogenesis and Tumor Growth The DAKO LSAB Kit for mouse and rabbit primary antibody (DAKO, Glostrup, Denmark) was used. Each tumor tissue sample was fixed with $10 \%$ formalin for staining Ki-67 and VEGF (vascular endothelial growth factor) and fixed with Zinc solution containing $0.1 \mathrm{M}$ Tris buffer, $\mathrm{pH}$ $7.4,0.03 \mathrm{M} \mathrm{Ca}\left(\mathrm{C}_{2} \mathrm{H}_{3} \mathrm{O}_{2}\right)_{2}, 0.027 \mathrm{M} \mathrm{Zn}\left(\mathrm{C}_{2} \mathrm{H}_{3} \mathrm{O}_{2}\right)_{2}$, and $0.037 \mathrm{M}$ $\mathrm{ZnCl}_{2}$ for staining CD31, then embedded in paraffin and sectioned at $5-\mu \mathrm{m}$ thickness. Tissue sections were deparaffined in toluene, dehydrated in graded alcohols, and incubated with Proteinase K (Sigma, Tokyo) at $37^{\circ} \mathrm{C}$ for $20 \mathrm{~min}$ for CD31 and VEGF staining, and heated by microwave for $15 \mathrm{~min}$ in citric acid buffer for Ki-67 staining. After incubating with $3 \%$ hydrogen peroxidase in methanol, the sections were incubated with a monoclonal anti-mouse CD31 (BD Pharmingen, San Diego, CA, U.S.A.), a monoclonal anti-mouse Ki-67 (DAKO, Glostrup, Denmark) at a dilution of 1/50, and a polyclonal anti-mouse VEGF (Calbiochem, Cambridge, MA, U.S.A.) at a dilution of $1 / 20$ for overnight at $4{ }^{\circ} \mathrm{C}$. Sections were incubated with biotin-conjugated goat anti-rabbit immunoglobulin for VEGF and with biotin-conjugated goat 
anti-rat immunoglobulin for CD31 and $\mathrm{Ki}-67$ for $30 \mathrm{~min}$ in room temperature followed by washing in PBS for $10 \mathrm{~min}$. The sections were then incubated with peroxidase-conjugated streptavidin solution for $5 \mathrm{~min}$ followed by washing in PBS for $5 \mathrm{~min}$. Sections were stained with freshly prepared aminoethylcarbazole solution (CD31 and VEGF sections) or diaminobenzidine solution ( $\mathrm{Ki}-67$ sections) for $10 \mathrm{~min}$ followed by washing for $5 \mathrm{~min}$ in tap water. Next, sections were counterstained with hematoxylin. CD31, and VEGF sections were mounted with aqueous mounting medium. Ki-67 sections were dehydrated and mounted with Eukitt mounting medium.

Nuclei positivities for Ki-67 were determined by counting at least 1500 tumor cells at $400 \times$ magnification $(=0.07$ $\mathrm{mm}^{2}$ ). Vessel number was assessed by counting the four areas of greatest neovascularization that were identified at $200 \times$ magnification $\left(=0.13 \mathrm{~mm}^{2}\right)$, and vessel area $(\%)$ of the same field was morphometrically measured using WinRoof morphometry software (Sankou Co., Fukui). The intracellular VEGF immunostaining was assessed using a semi-quantitative scale $(-$ not detected; + moderate; ++ strong).

Statistical Analyses Data are presented as the mean standard deviation (S.D.). Statistically significant differences between the groups were determined with Student's twotailed $t$-test. All $p$-values are 2 -sided; values are considered statistically significant at $p<0.05$.

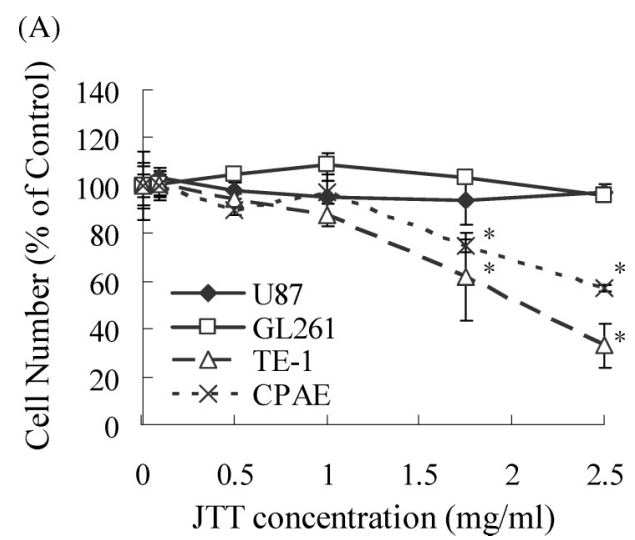

(B)

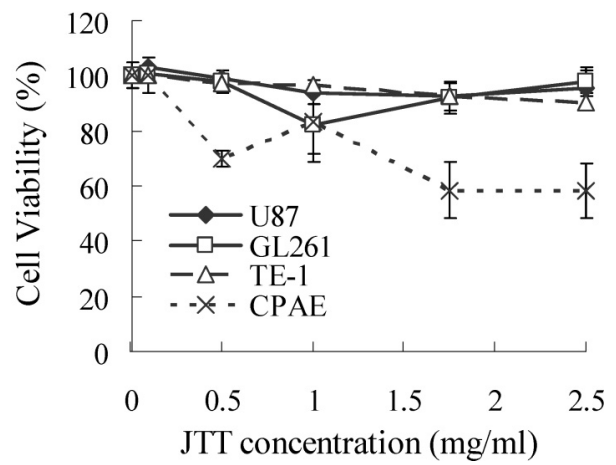

Fig. 1. Inhibition of Cell Proliferation (A) and Cell Viability (B) with JTT Extracts for Glioma Cells (U87 and GL261) and Endothelial Cells (TE-1 and CPAE)

The inhibition of endothelial cell proliferation (TE-1) was observed, but not glioma cell proliferation (U87 and GL261), at a concentration of $1.75 \mathrm{mg} / \mathrm{ml}$, without significant cell toxicity $(* p<0.05)$.

\section{RESULTS}

Effect of JTT on Cell Proliferation JTT extracts inhibited the endothelial cell (TE-1) proliferation but not glioma cell (U87 and GL261) proliferation at a concentration of 1.75 $\mathrm{mg} / \mathrm{ml}(p<0.05)$, without showing significant cell toxicity (Figs. 1A, B). However, CPAE cell viability was decreased at the same concentration. At lower concentrations of $0.1,0.5$, and $1 \mathrm{mg} / \mathrm{ml}$, JTT did not inhibit the proliferation both of glioma and endothelial cells.

JTT Treatment on GL261 Subcutaneous Growth and Weight Fluctuation After the tumor implantation, young and old mice were separated into three groups: high-dose JTT (young, $n=5$; old, $n=5$ ), low-dose JTT (young, $n=5$; old, $n=5$ ), and control (young, $n=5$; old, $n=5$ ). Mice in the JTT groups were fed the diet containing JTT for 3 weeks. One old mouse in the low-dose group expired because of a mechanical problem in the water supply. The volume of food intake was $3.8 \mathrm{~g} / \mathrm{d} /$ mouse in the control group and 3.6 $\mathrm{g} / \mathrm{d} /$ mouse in the JTT groups. No difference in food intake was observed between the control group and the JTT group (data not shown).

The tumor volume in each group is shown in Fig. 2. There was no significant difference in the tumor volume between the control group and the JTT group in either young mice (A) or old mice (B). The weight loss in the JTT treatment groups was inhibited in a dose-dependent manner in each age group. The body weight of old mice with high dose JTT was significantly higher compared to control mice, suggesting JTT influenced good health condition especially for old mice $(p<0.05)$ (Table 1).
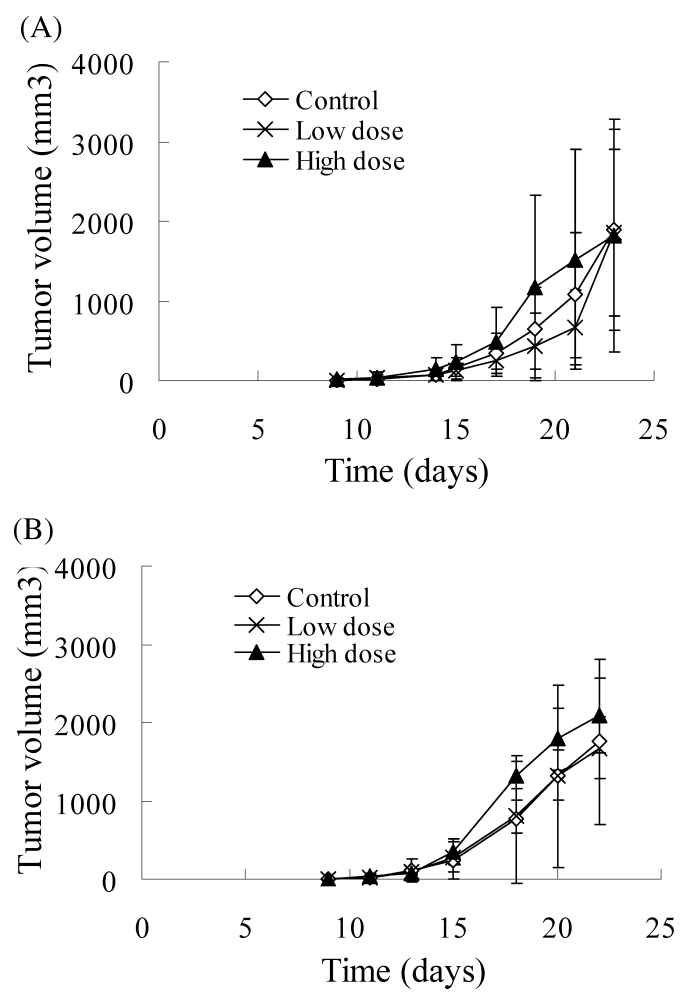

Fig. 2. Tumor Volume $\left(\mathrm{mm}^{3}\right)$ after JTT Administration in GL261-Bearing C57BL/6J Young (A) and Old (B) Mice

Mice were fed with a control diet or with a low dose $(500 \mathrm{mg} / \mathrm{kg})$ or high dose $(1000$ $\mathrm{mg} / \mathrm{kg}$ ) of JTT. Tumor volume in the groups was not different during the experiment. 
Table 1. Changes of Body Weight with/without JTT Treatment

\begin{tabular}{llc}
\hline \hline \multicolumn{1}{c}{ Groups } & $\begin{array}{c}\text { Change of body } \\
\text { weight }(\mathrm{g})\end{array}$ \\
\hline \multirow{2}{*}{ Young } & Control $(n=5)$ & $+1.6 \pm 0.5$ \\
& Low dose $(n=5)$ & $+2.4 \pm 1.1$ \\
& High dose $(n=5)$ & $+3.8 \pm 3.6$ \\
Old & Control $(n=5)$ & $0.4 \pm 0.5$ \\
& Low dose $(n=4)$ & $0 \pm 1.6$ \\
& High dose $(n=5)$ & $+1.3 \pm 0.5^{*}$ \\
\hline
\end{tabular}

$* p<0.05$ compared with control by Student's two tailed $t$-test.

Table 2. Surface Phenotypic Analysis of Spleen Lymphocytes with/without JTT Treatment

\begin{tabular}{clcccc}
\hline \hline \multicolumn{1}{c}{ Groups } & $\begin{array}{c}\text { NK cells } \\
(\%)\end{array}$ & $\begin{array}{c}\text { NKT cells } \\
(\%)\end{array}$ & $\begin{array}{c}\text { T cells } \\
(\%)\end{array}$ & $\begin{array}{c}\text { CD4/CD8a } \\
\text { ratio }\end{array}$ \\
\hline \multirow{2}{*}{ Young } & Control $(n=4)$ & $1.97 \pm 0.52$ & $0.95 \pm 0.23$ & $29.3 \pm 0.86$ & $1.59 \pm 0.19$ \\
& Low dose $(n=4)$ & $2.12 \pm 0.46$ & $0.91 \pm 0.42$ & $30.3 \pm 2.69$ & $1.45 \pm 0.13$ \\
& High dose $(n=4)$ & $2.06 \pm 0.42$ & $0.96 \pm 0.25$ & $31.2 \pm 2.95$ & $1.39 \pm 0.14$ \\
\multirow{6}{*}{ Old } & Control $(n=4)$ & $1.88 \pm 0.14$ & $1.88 \pm 0.34$ & $28.1 \pm 2.65$ & $2.12 \pm 0.59$ \\
& Low dose $(n=4)$ & $2.26 \pm 0.42$ & $1.24 \pm 0.30$ & $24.7 \pm 5.09$ & $2.11 \pm 0.36$ \\
& High dose $(n=4)$ & $2.46 \pm 0.34 *$ & $1.58 \pm 0.36$ & $23.4 \pm 1.38$ & $2.40 \pm 0.13$ \\
\hline
\end{tabular}

$* p<0.05$ compared with control by Student's two tailed $t$-test.

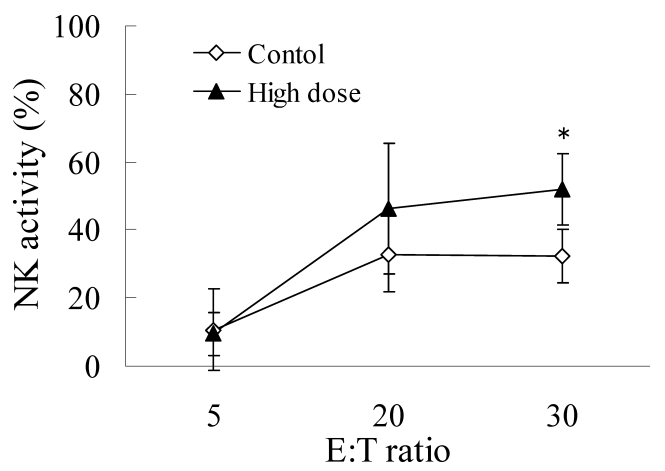

Fig. 3. NK Activity between the JTT High-Dose Group and the Control Group of Old Mice

YAC-1 cells at $\left(1 \times 10^{5}\right) /$ well were incubated with fresh nonactivated spleen cells in fixed $\mathrm{E}: \mathrm{T}$ ratios for $4 \mathrm{~h}$, and NK activity was measured by the modified WST-8 assay. Enhancement of NK activity was observed in the high-dose group of old mice $(* p<0.05)$

Effect of JTT Treatment on the Immunological Functions Mice were fed the diet containing JTT for three weeks after tumor implantation. Spleens were obtained from four mice in each group. The ratio of NKT cells (CD3+ $\mathrm{NK} 1.1+$ cells), and $\mathrm{T}$ cells (CD3+NK1.1-cells), and the $\mathrm{CD} 4 / \mathrm{CD} 8$ ratio in $\mathrm{T}$ cells between any of the groups did not differ significantly in either young or old mice. On the other hand, the ratio of NK cells (CD3-NK1.1+ cells) increased in a dose-dependent manner with JTT administration in the old mice, but not in the young mice (Table 2). Because of this, we evaluated NK activity in the old mice. We found that NK activity significantly increased to an $E$ : T ratio of $30: 1$ in the JTT-treated old mice compared to the control mice $(p<0.05$, Fig. 3). Data were presented as the mean of three wells in each sample.

Immunohistochemistry for CD31, VEGF, and Ki-67
Table 3. Vascular and Proliferative Characteristics of Tumors with/without JTT Treatment

\begin{tabular}{clcccc}
\hline \hline Groups & $\begin{array}{c}\text { Vessel } \\
\text { numbers/ } \\
0.13 \mathrm{~mm}^{2}\end{array}$ & $\begin{array}{c}\text { Vessel } \\
\text { area } \\
(\%)\end{array}$ & $\begin{array}{c}\text { Ki-67 } \\
\text { positivities } \\
(\%)\end{array}$ & $\begin{array}{c}\text { VEGF } \\
\text { staining }\end{array}$ \\
& & & & \\
\hline \multirow{2}{*}{ Young } & Control $(n=5)$ & $13.7 \pm 2.75$ & $5.71 \pm 2.33$ & $71.2 \pm 5.40$ & ++ \\
& Low dose $(n=5)$ & $13.9 \pm 7.65$ & $3.13 \pm 1.31$ & $74.1 \pm 7.43$ & ++ \\
& High dose $(n=5)$ & $13.2 \pm 4.75$ & $2.84 \pm 0.85$ & $72.0 \pm 2.78$ & ++ \\
Old & Control $(n=5)$ & $13.1 \pm 4.60$ & $4.38 \pm 1.24$ & $74.4 \pm 4.01$ & ++ \\
& Low dose $(n=4)$ & $12.5 \pm 2.27$ & $2.72 \pm 0.82 *$ & $72.8 \pm 2.75$ & ++ \\
& High dose $(n=5)$ & $11.7 \pm 4.29$ & $1.89 \pm 0.44 *$ & $70.8 \pm 1.84$ & ++
\end{tabular}

a) ++ was VEGF strongly positive. $* p<0.05$ compared with control by Student's two tailed $t$-test.

The immunohistochemical results are summarized in Table 3 . Vessel number and area and VEGF positivities were evaluated for the degree of angiogenesis and Ki-67 for the tumor proliferative potential within the tumor tissues. As shown in Fig. 4, control tumors were highly vascularized with large lumens. In contrast, prominent small vessels were observed in the JTT-treated groups. Vessel number was not changed between the groups, but vessel area was significantly decreased in the JTT-treated old mice in a dose-dependent manner (control $4.38 \pm 1.24 \%$; low dose $2.72 \pm 0.82 \%$; high dose $1.89 \pm$ $0.44 \%, p<0.05)$. However, $\mathrm{Ki}-67$ of glioma cells and VEGF positivities in the tumor tissues were similar between the control group and treatment groups.

\section{DISCUSSION}

In the present study, we demonstrated that JTT had antiangiogenic as well as immuno-biological activities on malignant gliomas, but only in old mice. JTT treatment resulted in the decrease of vessel area within the tumor tissues in vivo and of endothelial cell proliferation in vitro.

JTT has been reported to show anti-tumor effects through the reduction of metastatic potential and enhancement of cytokine induction for various kinds of solid tumors, including gliomas. ${ }^{4,6,8)}$ Takahashi has reported that JTT administration for 4 weeks inhibited the subcutaneous growth of human and mouse gliomas, and enhanced the survival rate of tumorbearing mice, possibly through the activation of natural killer (NK) cells and tumor necrosis factor (TNF) in the immune system. ${ }^{5,17)}$ The anti-tumor mechanism of JTT appears in part to involve the ability to induce endogenous TNF production and NK cell activity. ${ }^{4,18)}$ However, while there were many reports of anti-tumor effects of JTT, there were some reports that it was not effective or only slightly effective in inhibiting the growth of tumor with JTT alone. Nakamura et al. has reported that the combined effect of JTT and 1-(4-amino-2methyl-5-pyrimidinyl)methyl-3-(2-chloroethyl)-3-nitrosourea (ACNU), a cytotoxic agent commonly used in the treatment of malignant gliomas, enhanced the inhibitory effect of mouse glioma growth, although it was not effective with JTT alone. $^{19)}$

Clinically, JTT is known to improve the general systemic condition of cancer patients and to reduce the adverse effects of chemotherapy, radiation therapy, and surgical treatment. ${ }^{4}$ Immunological function was monitored in patients with brain tumors receiving JTT orally, and Miyagami and Katayama ${ }^{9)}$ 

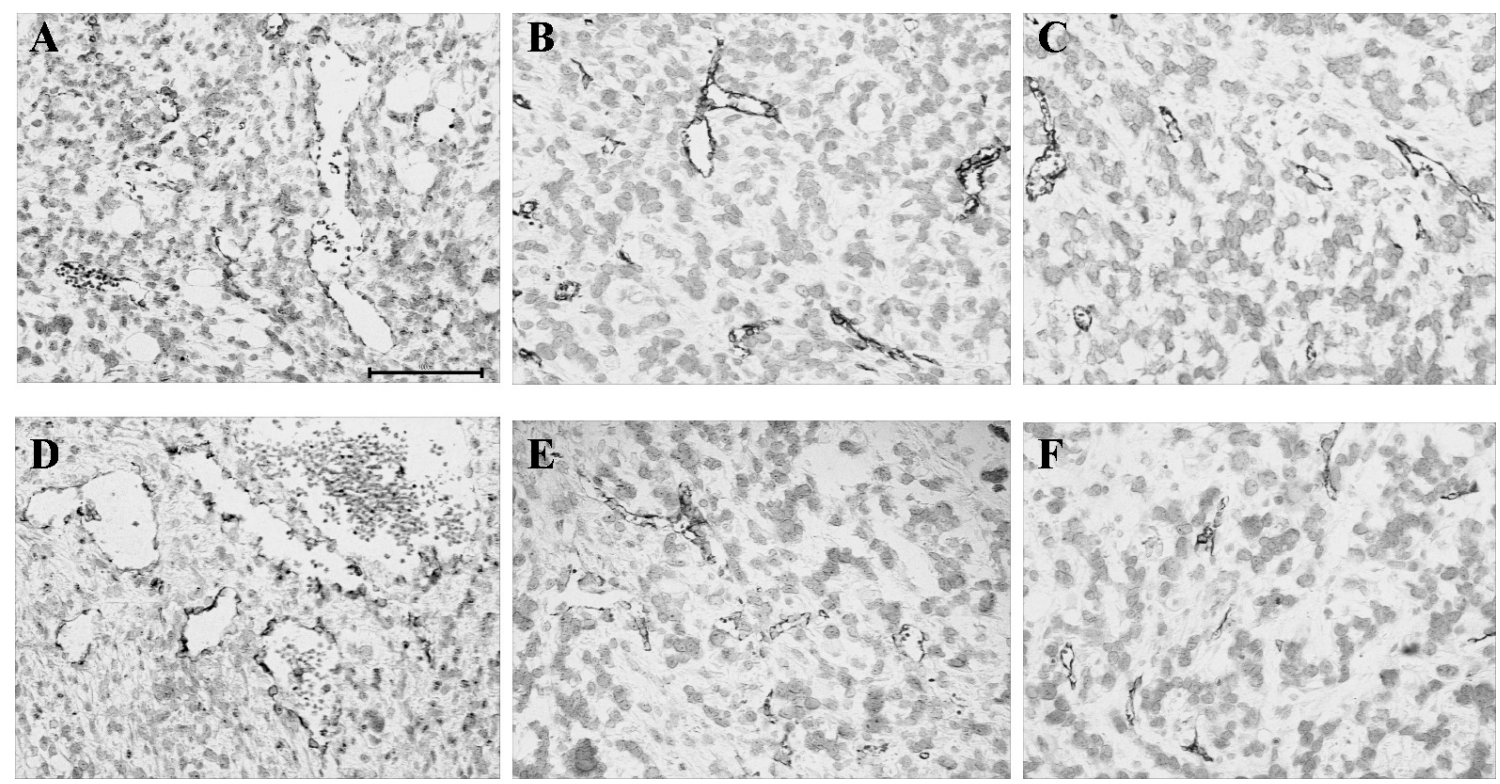

Fig. 4. Morphometrical Analysis of the Vessel Area of GL261 Tumors from Control and JTT-Treated Mice at $200 \times$ Magnification $\left(=0.13\right.$ mm $\left.^{2}\right)$

Tumor sections were immunostained with anti-CD31 antibody. (A) Young mice (control group), (B) young mice (low-dose JTT group), (C) young mice (high-dose JTT group), (D) old mice (control group), (E) old mice (low-dose JTT group) and (F) old mice (high-dose JTT group). Control tumors were highly vascularized with large lumens. In contrast, prominent small vessels were observed in the JTT-treated groups. Bars, $100 \mu \mathrm{m}$.

reported that JTT administration resulted in (1) a decrease of suppressor T cells, (2) an increase of TNF- $\alpha$ productivity, (3) activation of NK cells combined with interferon- $\beta$ treatment, and (4) no change of cytotoxic T cell or NK activity. In our results, we did not observe the inhibition of glioma growth by JTT administration in GL261-bearing C57BL/6J young and old mice. However, the NK cell ratio within PBMC and the NK activity of fresh splenocytes obtained from JTTtreated old mice were significantly increased. We could not only confirm that JTT administration improved the host immune function of experimental animals with glioma, but we could also demonstrate that the factor of age is important for its activity.

The level of immunological functions of old mice was lower than that of young mice. The age-related decline of immunological functions mainly occurs in T cell- and NK celldependent immunity. ${ }^{20)}$ However, the decline of immunological functions can be restored to the level seen in young mice by the administration of herbal medicines. Among more than one hundred kinds of herbal medicines, JTT is well known to enhance the immunological functions. ${ }^{8,9)}$

There are only few reports that describe the relationship between age and immunological functions. Utsuyama et al. have reported the effect of JTT on immunological functions in young and old mice. ${ }^{10)}$ In their experiments, a significant increase in the number of $\mathrm{T}$ cells and NK cells was observed in JTT treatment of old mice, and NK activity was definitely enhanced in JTT treatment of old mice. We demonstrated the increase of NK cell ratio and the enhancement of NK activity by JTT administration in old tumor-bearing mice. Tumor bearing could be a miserable stress for small animals, such as mice. In fact, control tumor-bearing mice in our experiment lost their body weight during the experiment. JTT-treated mice restored their body weight during the experiment. JTT is useful for increasing the immunological functions, especially in old mice. These facts clearly indicate the importance of age when observing the effects of herbal medicines.

Another important finding in our experiment is the antiangiogenic effect of JTT. There are very limited reports of anti-angiogenic studies using crude extracts or oral administration of herbal medicines. The boiling water extracts of 24 herbal medicines usually used to cure ischemic disease were screened for angiogenic and anti-angiogenic effect, respectively. ${ }^{14)}$ These effects were assessed by quantification of vessels on chick embryo chorioallantoic membrane (CAM) assay and cell proliferation assay of cultured bovine aortic endothelial cells (BAECs). Although some of the extracts showed angiogenic effects, 6 crude extracts (Berberis paraspecta, Catharanthus roseus, Coptis chinensis, Polygonum cuspidatum, Scrophularia ningpoensis, and Scutellaria baicalensis) showed a strong anti-angiogenic effect. However, JTT do not contain one of the extracts shown to have a strong anti-angiogenic effect. In the anti-angiogenic experiment of oral administration of herbal medicine, the anti-angiogenic effect of Qing-Luo-Yin (QLY), a herbal medicine for the treatment of rheumatoid arthritis, was investigated in a rat model of collagen-induced arthritis. ${ }^{13)}$ QLY administration inhibited the over-expression of matrix metalloproteinases-3, which plays an important role in the migration of endothelial cells, and it enhanced the tissue inhibitors of metalloproteinases. We observed the decrease of vessel area in tumor tissues treated by JTT administration, especially in old mice. In addition, the endothelial cell proliferation (TE1) was inhibited by JTT extract.

It is important to know about the anti-angiogenic effect with JTT administration, because good candidate for anti-angiogenic drugs include those available for oral administration for a long term without severe systemic side effects. ${ }^{21)}$ JTT has satisfied these conditions and could be a candidate for an anti-angiogenic material for patients with malignant gliomas.

Our experiment showed the anti-angiogenic effect and the enhancement of immunity with JTT administration. In the re- 
lationship between angiogenesis and immunological function, it has been reported that VEGF regulates NK cell adhesion to tumor endothelium. ${ }^{11)}$ Furthermore, IFN- $\gamma$ produced by activated NK cells decreased blood vessel densities in tumor tissue. ${ }^{12)}$ The enhancement of immunological function by JTT administration could be related to the suppression of angiogenesis in our experiment. However, we did not observe the inhibition of VEGF produced by tumors in the JTT-administered mice. It would be helpful to demonstrate the adhesion of NK cells activated by JTT in a future study.

Our result indicates that a decrease in vessel area in JTT treated mice did not affect the glioma growth. Therefore, the main question would be the biological significance of the anti-angiogenic effect, including the decreases of vessel area, to totally inhibit well-establish tumors. The supply of oxygen and nutrition to the tumor is different according to the size of the blood vessel. Tropres et al. has indicated that the vessel size imaging was a quantitative method for tumor vasculature characterization. $^{22)}$ Therefore, we considered that it is important for tumor angiogenesis to prevent not only the number of blood vessel but also the area of blood vessels. On the other hand, faster growing tumor occasionally does not shrink during various anti-angiogenic therapies alone. ${ }^{23)}$ Preclinical studies have shown that faster growing tumors may need a proper adjustment of the anti-neoplasmic drug and the drug schedule. $^{24)}$ These reports suggest that it is necessary for rapidly strong tumor, such as malignant glioma, to devise the use of anti-angiogenic drugs, such as in combination with other natural or synthetic anti-neoplasmic drug in a strategy of the anti-angiogenic therapy.

In conclusion, JTT exerted an anti-angiogenic effect and enhancement of the immunological function on malignant gliomas, especially in old mice. JTT would be useful as an adjuvant medicine for malignant gliomas through its dual function of enhancing systemic immunological function and exerting an anti-angiogenic action.

Acknowledgements We gratefully acknowledge Dr. Tsutomu Kaneko and Dr. Toshitaka Kido (Kampo and Pharmacognosy Laboratory, Tsumura) for FACS analysis and useful discussion, and Ms. Makiko Miyakawa and Yoshiko Tsukada for their excellent technical assistance. This work was supported by a Grant-in-Aid for Scientific Research from the Japanese Ministry of Education, Culture, Sports, Science and Technology, a grant from Kampo Fundation, and a grant from the Tsukuba Advanced Research Alliance.

\section{REFERENCES}

1) Takano S., Kamiyama H., Tsuboi K., Matsumura A., Brain Tumor Pathol., 21, 69-73 (2004).

2) Takano S., Tsuboi K., Matsumura A., Nose T., Nuro-Oncol., 5, 1-7 (2003).

3) Borchers A. T., Hackman R. M., Keen C. L., Stern J. S., Gershwin M. E., Am. J. Clin. Nutr., 66, 1303-1312 (1997).

4) Saiki I., Biol. Pharm. Bull., 23, 677-688 (2000).

5) Takahashi H., The Japan Infant Oriental Medicine Workshop Bulletin, 13, 5-13 (1997).

6) Ohnishi Y., Fujii H., Hayakawa Y., Yamamura T., Sakamoto T., Tsukada K., Fujimaki M., Nunome S., Komatsu Y., Saiki I., Jpn. J. Cancer Res., 89, 206-213 (1998).

7) Saiki I., Yamamura T., Ohnishi Y., Hayakawa Y., Komatsu Y., Nunome S., Chem. Pharm. Bull., 47, 1170-1174 (1999).

8) Matsumoto T., Sakurai M. H., Kiyohara H., Yamada H., Immunopharmacol., 46, 149-161 (2000).

9) Miyagami M., Katayama Y., No Shinkei Geka, 31, 401-409 (2003).

10) Utsuyama M., Seidlar H., Kitagawa M., Hirokawa K., Mech. Ageing Dev., 122, 341-352 (2001).

11) Melder R. J., Koenig G. C., Witwer B. P., Safabakhsh N., Munn L. L., Jain R. K., Nat. Med., 9, 992-997 (1996).

12) Hayakawa Y., Takeda K., Yagita H., Smyth M. J., Van Kaer L., Okumura K., Saiki I., Blood, 100, 1728-1733 (2002).

13) Li S., Lu A. P., Wang Y. Y., Li Y. D., Am. J. Chin. Med., 31, 713-720 (2003).

14) Wang S., Zheng Z., Weng Y., Yu Y., Zhang D., Fan W., Dai R., Hu Z., Life Sci., 74, 2467-2478 (2004).

15) Tanaka M., Tanaka T., Harata M., Suzuki T., Mitsui Y., Biochem. Biophys. Res. Commun., 243, 531-537 (1998).

16) Ribeiro-Dias F., Marzagao Barbuto J. A., Tsujita M., Jancar S., J. Immunol. Methods, 241, 121-129 (2000).

17) Takahashi H., Kanpo to Saishinchiryou, 2, 133-137 (1995).

18) Haranaka K., Satomi N., Sakurai A., Haranaka R., Kosoto H., Kobayashi M., Gendai Touyou Igaku, 6, 85-91 (1985).

19) Nakamura O., Okamoto K., Kaneko M., Hirohiko H., Shitara N., Biotherapy, 8, 1003-1006 (1994).

20) Kutza J., Kaye D., Murasko D. M., J. Gerontol. A. Biol. Sci. Med. Sci., 4, B110-116 (1995).

21) Kerbel R., Folkman J., Nat. Rev. Cancer, 2, 727-739 (2002).

22) Tropres I., Lamalle L., Peoc'h M., Farion R., Usson Y., Decorps M., Remy C., Magn. Reson. Med., 51, 533-541 (2004).

23) Carmelit P., Jain R. K., Nature (London), 407, 249-257 (2000).

24) Bisacchi D., Benelli R., Vanzetto C., Ferrari N., Tosetti F., Albini A., Cancer Detect. Prev., 27, 229-238 (2003). 